\title{
Influence of bisphosphonates on alveolar bone density: a histomorphometric analysis
}

\author{
Milton Santamaria Júnior ${ }^{(a)}$ \\ Ana Carolina Cuzzuol Fracalossi ${ }^{(b)}$ \\ Maria Fernanda Martins Ortiz \\ Consolaro(a) \\ Alberto Consolaro(c) \\ (a) $\mathrm{PhD}$ in Oral Pathology; (b) MS in Oral \\ Pathology; (c) Full Professor of Oral \\ Pathology, Department of Oral Pathology \\ - Bauru Dental School, University of São \\ Paulo, Bauru, SP, Brazil.
}

\begin{abstract}
This study is a histomorphometrical analysis of the influence of the bisphosphonate alendronate on alveolar bone density. Eighteen male Wistar rats were randomly assigned to a control group $(\mathrm{n}=9)$ that received no medication and an experimental group $(n=9)$ that received oral alendronate $(1 \mathrm{mg} / \mathrm{kg})$ from birth until euthanization at 3 months of age. Semi-serial 4- $\mu \mathrm{m}$-thick transverse sections were obtained from the region between the roots of the left maxillary first molar, stained with hematoxylin and eosin, and examined with a Zeiss Axioskop II optical microscope for histomorphometric analysis. The images were captured with a digital camera coupled with the microscope and connected to a computer, and were analyzed using Image J $1.34 \mathrm{~s}$ image-analysis software. A 1,200-point grid was positioned onto each digitized image. The number of intersection points of grid lines in the bone tissue was counted. The ratio between the number of points in the bone tissue and the total number of points of the grid $(1,200)$ was used to determine the bone density of the analyzed tissue. Data from the control and experimental groups were compared and analyzed statistically by the Student's t-test $(\mathrm{p}=0.05)$. There was no statistically significant difference $(\mathrm{p}=0.3754)$ in the alveolar bone density between the control and alendronate-treated animals. It may be concluded that the bisphosphonate alendronate did not alter the morphology of the alveolar bone, maintaining its structural tissue characteristics in healthy animals.
\end{abstract}

Descriptors: Diphosphonates; Alendronate; Bone density; Alveolar bone loss.

\section{Introduction}

Pyrophosphates (P-O-P), bisphosphonates precursors, have been known to chemists since the $19^{\text {th }}$ century, the first synthesis dating back to 1865 in Germany. ${ }^{1}$ The initial uses for pyrophosphates and bisphosphonates in industry were as antiscaling and anticorrosive agents, emollients for the prevention of calcium carbonate precipitation in metallic plumbing. An expanded understanding of bone physiology, as well as a growing appreciation of bisphosphonate chemistry, allowed a broadening range of clinical applications. Further research showed that bisphosphonates were effective in controlling calcium phosphate formation and dissolution in vitro as well as bone resorption and mineralization in vivo, extending its therapeutic use for the treatment of innumerous diseases.
Received for publiction on Mar 16, 2010 Accepted for publication on May 03, 2010 
Pyrophosphates are physiological regulators of calcification and bone resorption, which are naturally present in the serum and urine. Bisphosphonates are structural analogs of pyrophosphates and their mechanism of action is similar to that of the aforementioned compounds. ${ }^{1}$

The discovery that bisphosphonates have potent inhibitory effects on bone resorption occurred in the 1960 's, ${ }^{2,3}$ after several attempts to identify agents that were pyrophosphate analogs and could also regulate bone calcification, thus being potentially useful for the prevention of osteoporosis, arthritis, osteolytic diseases and bone metastases resulting from malignant neoplasias.

Alendronate, clodronate, pamidronate and zoledronate are some of the bisphosphonates used for the treatment of bone diseases. ${ }^{4,5}$ Each drug presents a peculiar physicochemical structure and specific biological functions, which mean that the results of experimental evaluations obtained for one bisphosphonate cannot be extrapolated to another. ${ }^{4}$ The use of bisphosphonate drugs by patients with Paget's disease decreases bone turnover, reducing bone loss. ${ }^{4,5}$ The administration of bisphosphonates to patients with metastases has been shown to significantly reduce the occurrence of bone fractures, malignant hypocalcaemia, need of radiotherapy and bone surgery due to neoplasic tumors. ${ }^{6,7,8}$

Bisphosphonates that contain nitrogen, such as alendronate, have been shown to be more potent inhibitors of bone resorption in vivo than those that do not have this chemical element in their composition. ${ }^{9}$ These nitrogen-containing bisphosphonates reduce the recruitment of clastic cells and induce osteoblasts to produce an inhibitory factor to osteoclast formation. ${ }^{10,11}$ In addition, the action of alendronate induces osteoclast apoptosis and reduces the participation of these cells in bone resorption (Figure 1). Alendronate is widely used in medicine in the control of osteopenia, preventing osteoporosis and the occurrence of fractures in women with postmenopausal osteoporosis..$^{12,13}$

The use of bisphosphonates has increasingly gained the attention of dental professionals. The clinical protocols for the treatment of dental patients under bisphosphonate therapy in order to pre- vent the occurrence of postoperative complications, such as osteomyelites, ${ }^{8}$ after surgical periodontal procedures, extractions or implant placement, and delay of tooth movement during orthodontic treatment has been discussed in literature. Older patients are more commonly seeking dental offices with both functional and esthetic demands. Many of these patients, however, have systemic conditions that require long-term use of certain medications, such as bisphosphonates. It is certainly not uncommon that postmenopausal female patients undergoing therapy with oral alendronate, for example, attend dental offices without the dentist being made aware of their drug therapy.

It is possible that the prolonged use of alendronate increases the alveolar bone density and this structural alteration could play a role in reducing the repair capacity of the alveolar bone tissue in response to different dental procedures. Therefore, this study histomorphometrically analyzed the influence of alendronate on alveolar bone density in the maxilla of rats treated with this bisphosphonate.

\section{Material and Methods}

Eighteen 90-day-old male Wistar rats (Rattus norvegicus albinus) with initial mean weight of $300 \mathrm{~g}$ were obtained from the vivarium of Bauru Dental School, São Paulo State University, Brazil af-

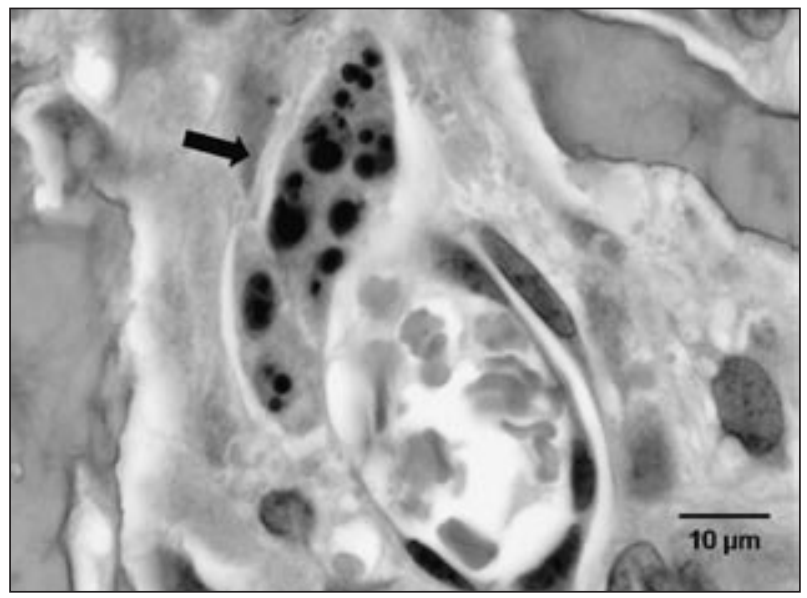

Figure 1 - Osteoclast in apoptosis (arrow) close to a congested vessel found in some animals of the alendronatetreated group. Note the chromatin condensation and loss of nuclear polarization (Hematoxylin and eosin - HE; $\times 1000$ magnification). 
ter approval of the research protocol by the institutional Animal Care and Research Use Committee. All guidelines regarding the care of animal research subjects were strictly followed in this study. The animals were housed in cages and maintained in a clean, ventilated environment with natural lighting and a mean temperature of $25^{\circ} \mathrm{C},{ }^{14}$ and were fed standard rat chow (Guabi; Nutrilabor, Campinas, SP, Brazil) and water ad libitum during the entire experimental period.

The animals were randomly assigned to a control group $(\mathrm{n}=9)$, which did not receive any medication, and an experimental group $(\mathrm{n}=9)$ to which oral sodium alendronate (Alendil; Farmoquímica, Rio de Janeiro, RJ, Brazil) was administrated twice a week ${ }^{15}$ at the same time of day with 3 day intervals between the doses. The bisphosphonate was administered to the rats from birth until euthanization. The drug was fractionated to obtain a dose compatible to the dose given to human patients $(1 \mathrm{mg} / \mathrm{kg}$ ) and was administered by gavage as a solution using syringes coupled with $7 \mathrm{~cm}$ urethral catheters. The cannula of the catheter was easily introduced through the throat of the rat without the risk of regurgitation of the medication.

The animals were euthanized at 3 months of age with a lethal injection of a mixture of ketamine hydrochloride (Dopalen - $100 \mathrm{mg} / \mathrm{ml}$; Vetbrands, Jacareí, SP, Brazil) and xylazine hydrochloride (Anasedan - 20 mg/ml; Vetbrands, Jacareí, SP, Brazil), followed by decapitation and dissection of the epithelial and muscular tissues circumjacent to the maxilla. The maxillas were fixed in buffered $10 \%$ formalin within 48 hours of the euthanization of the animals, demineralized in Morse solution (equal volumes of sodium solution and formic acid) within 7 days of the euthanization of the animals and embedded in paraffin. Semi-serial 4- $\mu \mathrm{m}$-thick transversal sections were obtained, stained with hematoxylin and eosin, and examined with a Zeiss Axioskop II optical microscope (Carl Zeiss AG, Oberkochen, Germany) with a $\times 10$ magnification objective lens (Carl Zeiss AG, Oberkochen, Germany) for histomorphometric analysis. The medullar alveolar bone between the roots of the left maxillary first molar at the cervical third was evaluated. The images were captured with a digital camera (CCD IRIS/RGB, Sony Corp., Tokyo, Japan) coupled with the microscope and connected to a computer and were analyzed using Image J 1.34s image-analysis software (National Institute of Health, Bethesda, Maryland, USA). A 1,200-point grid with a total area of $694 \times 520 \mu \mathrm{m}$ was positioned onto each digitized image. The number of intersection points of the horizontal and vertical lines of the grid on the image of the bone tissue (not in the medullar spaces) was counted. The ratio between the number of points in the bone tissue and the total number of points of the grid $(1,200)$ allowed for the determination of the bone density of the analyzed tissue (Figure 2).

The bone density data from the control and alendronate-treated groups were compared and statistically analyzed by the Student's t-test at 5\% significance level using SAS software (v.3.43; SAS Institute Inc., Cary, North Carolina, USA).

\section{Results}

The mean bone density values for each animal and for each group are represented in Table 1. There was no statistically significant difference $(\mathrm{p}=0.3754)$ in the alveolar bone density between the control and alendronate-treated animals (Table 1 and Graph 1). The groups were homogeneous with an $\mathrm{F}=0.89$ (homogeneity is considered if $\mathrm{F}>0.05$ ).

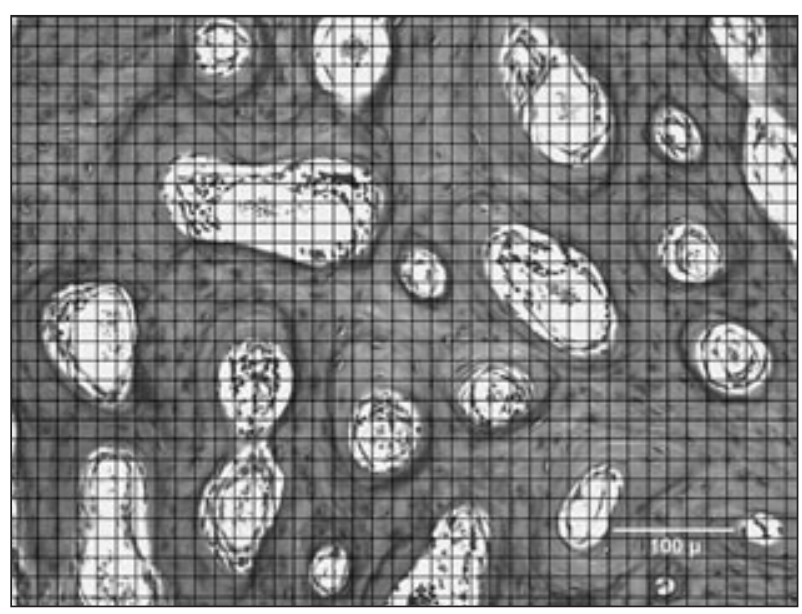

Figure 2 - 1,200-point grid positioned onto each digitized image of a histological section. The number of intersection points of the horizontal and vertical lines of the grid in the bone tissue (not in the medullar spaces) was counted. (Hematoxylin and eosin - HE; $\times 100$ magnification). 
Table 1 - Mean bone density values (bone areas/non-bone areas ratio) in each animal and each group.

\begin{tabular}{c|c|c|c|c|c|c|c|c|c|c}
\hline Group & 1 & 2 & 3 & 4 & 5 & 6 & 7 & 8 & 9 & Total \\
\hline Control & 92.22 & 81.43 & 85.31 & 72.21 & 77.10 & 76.86 & 83.56 & 59.49 & 83.28 & $79.05 \pm 9.31$ \\
\hline Alendronate & 73.70 & 61.87 & 79.96 & 84.67 & 82.08 & 61.94 & 79.30 & 64.80 & 86.24 & $74.95 \pm 9.75$ \\
\hline
\end{tabular}

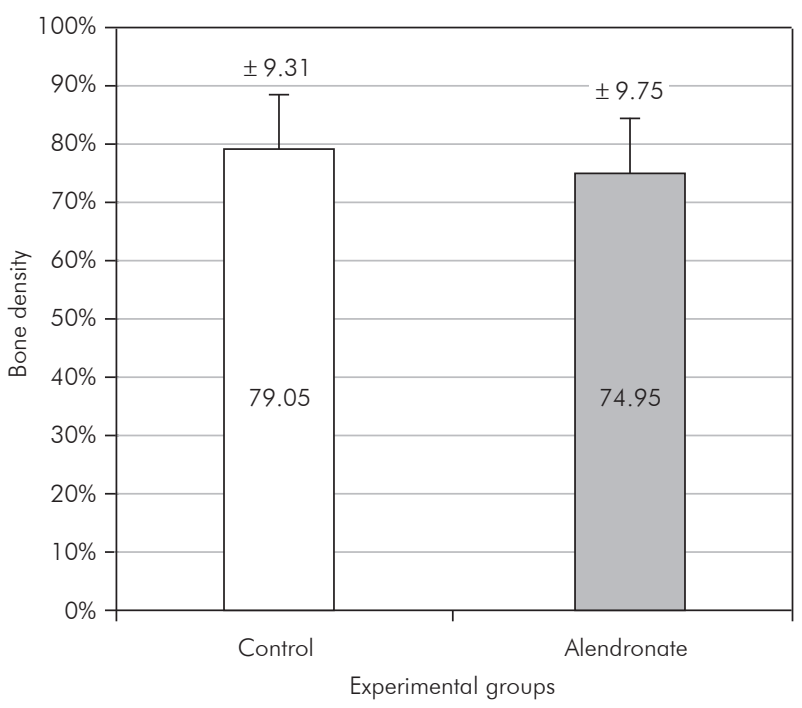

Graph 1 - Mean values of bone density and standard deviations for the control and alendronate-treated groups. Bone density was determined as the ratio between the number of points in the bone tissue and the total number of points of the grid $(1,200) . p=0.3754$ (Student's t-test).

\section{Discussion}

The effects of the bisphosphonates occur at both tissue and cellular levels. ${ }^{4}$ At a tissue level, these compounds promote a decrease of bone turnover, ${ }^{4}$ as demonstrated by biochemical markers, ${ }^{16,17,18}$ showing a reduction in the extension of the bone resorption areas and the depth of the resorbed areas. At a cellular level, bisphosphonates act inhibiting the activity and recruitment of osteoclasts to the bone surface, inducing the apoptosis of these cells and altering the mineral exchange. ${ }^{4}$ The bisphosphonates incorporated into the bone are released in Howship's lacunae and internalized in the osteoclasts by endocytosis, affecting these cells directly. After this phenomenon, the osteoclasts lose their ruffled border and their cytoskeleton is progressively disrupted, inactivating the resorptive activity of these cells and inducing apoptosis (Figure 1). ${ }^{19}$ This process is due to the inhibition of the enzyme farnesyl diphosphate synthase, ${ }^{20,21}$ especially by the administration of nitrogen-containing bisphosphonates, such as alendronate. This enzyme is necessary for the prenylation of small GTPases, ${ }^{22}$ which are responsible for the signaling proteins that control morphology, cytoskeleton arrangement, ruffled borders, vesicle transportation and apoptosis of osteoclasts. ${ }^{23}$

It is known that the systemic effects of bisphosphonates are not immediate ${ }^{4}$ and that, in spite of their recognized inhibitory effect of calcification in vitro, a prolonged administration of these drugs is necessary to produce an effective inhibition of bone resorption.

Bisphosphonates are incorporated into mineralized structures. After bone uptake, the bisphosphonates are liberated again only when the bone in which they are deposited is resorbed. Under pathological conditions, alveolar bone loss is related to alterations in the normal balance between bone formation and resorption. Therapeutic agents that are capable of avoiding bone resorption and acceleration of bone deposition may act in preventing bone loss in patients with conditions such as periodontal disease.

The effects of bisphosphonates in controlling alveolar bone loss in advanced periodontal disease and their action in the inflammatory processes associated with periodontal breakdown have been investigated in rat experimental models. ${ }^{24}$ The effects of bisphosphonates in controlling alveolar bone loss in advanced periodontal disease and their action in the inflammatory processes associated with periodontal breakdown have also been examined with the induction of periodontal disease by Porphyromonas gingivalis $^{25}$ in monkeys ${ }^{26}$ and dogs. ${ }^{27}$ The results of these studies confirm that bisphosphonates were more effective preventing alveolar bone loss in animals with experimentally induced periodontal disease. When large bone tissue destruction was present, the groups treated with bisphosphonates exhibited less bone loss and a greater amount of remaining alveo- 
lar bone. However, the microscopic results showed no differences in the mandibles of the animals with little or no periodontal disease compared to the healthy control animals. In the same way, a previous study evaluating the post-extraction bone repair in rat teeth showed that the administration of dichloromethylene bisphosphonate (clodronate) inhibited the occurrence of rapid alveolar bone loss after extraction. This effect was observed exclusively in the surgical wounds and no differences were found between control and experimental animals in the intact bone areas. ${ }^{28}$

In this way, studies investigating the effects of bisphosphonates on inflammatory process and alveolar repair seem to demonstrate that these drugs do not microscopically alter the bone structure in healthy areas, corroborating the results of the present study in which no difference was found in the bone density of control and experimental animals (Table 1 and Graph 1). Bisphosphonates bind preferentially to bones that have high turnover rates, and their distribution in the bone tissue is not homogeneous. ${ }^{4,5}$ Therefore, it may be inferred that the incorporation of bisphosphonates to maxillary bones with no pathological processes would provide a longer period of time for a structural organization, without compromising or altering their architecture.
The analysis by light microscopy did not show any effects of the alendronate on the composition of the mineralized bone matrix in either the control or experimental groups. Morphologically, the characteristics of the bone tissue were similar in both groups with respect to the distribution and number of reversion lines, proportion of medullar spaces, bone trabeculae and cortical bone. Similar distribution and number of osteoclasts and bone remodeling units in the analyzed bone structures was also observed in both groups (Figure 3).

The therapeutic effects of bisphosphonates do not depend on the inhibition of normal bone remodeling, but is associated, rather, with pathological bone resorption or with regions with high bone turnover. ${ }^{29}$ Therefore, based on the findings of the present study, no complications in dental treatments, such as orthodontic movement, dental extractions or implant placement, are expected because these procedures are usually performed in healthy bone tissue. $^{16}$

In the present study, there was no difference in the bone density between the animals that received alendronate and the non-treated control animals. It was not possible to determine, using optical microscopy, any effect of the drug in the composition of the mineralized bone matrix in either of the groups. Morphologically, the alveolar bone in the animals
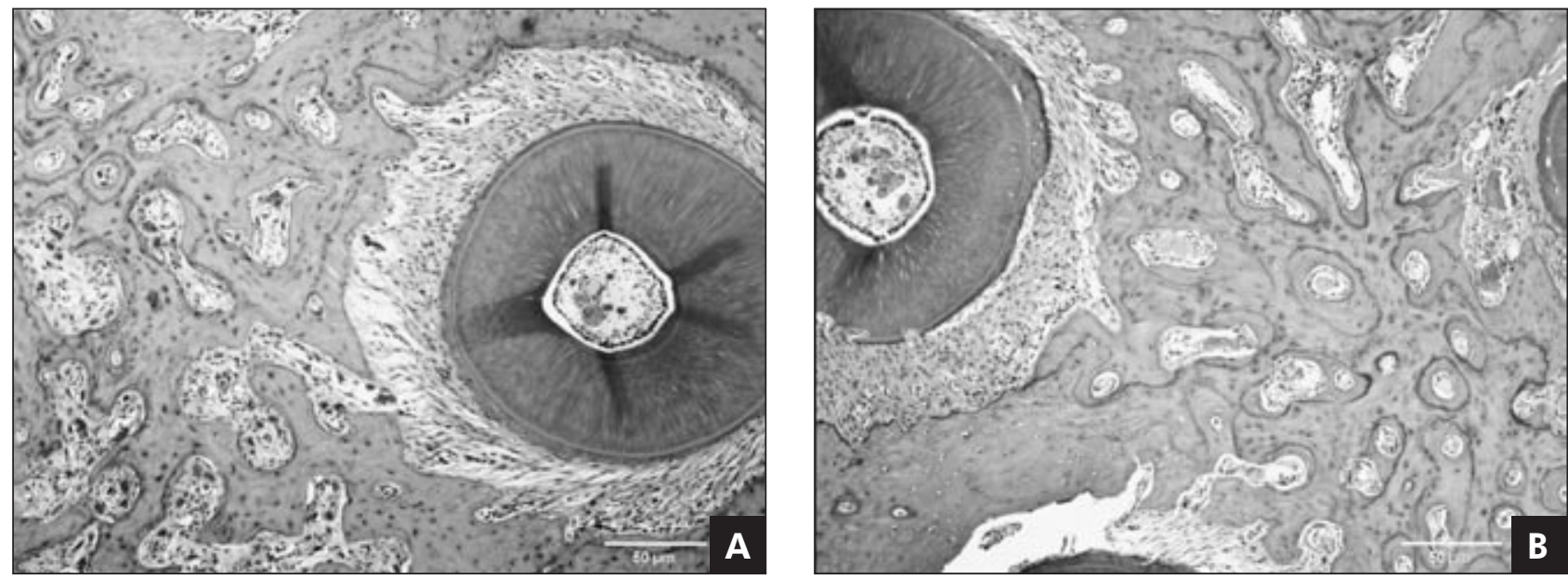

Figure 3 - Alveolar bone in the alendronate-treated (A) and control (B) group. The bone tissue characteristics were similar in both groups with respect to the distribution and number of reversion lines, and proportion of medullar spaces, bone trabeculae and cortical bone. The distribution and number of osteoclasts and bone remodeling units in the analyzed bone structures was also similar in both groups (Hematoxylin and eosin - $\mathrm{HE} ; \times 40$ magnification). 
treated with alendronate had the same proportions of medullar spaces and bone trabeculae as those of the control animals.

\section{Conclusion}

No statistically significant difference in alveolar bone density between the animals that received bisphosphonate (alendronate) and control animals was found.

Histomorphometric analysis using an optical microscope was unable to determine any effect of sodium alendronate in the composition of mineralized bone matrix between the control and experimental groups. Morphologically, the bone in the experimental and control groups showed comparatively

\section{References}

1. Fleisch H, Russell RG, Straumann F. Effect of pyrophosphate on hydroxyapatite and its implication in calcium homeostasis. Nature. 1966 Nov 26; 212(5065):901-3.

2. Bisaz S, Russell RG, Fleisch H. Isolation of inorganic pyrophosphate from bovine and human teeth. Arch Oral Biol. 1968 Jun;13(6):683-96.

3. Fleisch H, Russell RG, Francis MD. Diphosphonates inhibit hydroxyapatite dissolution in vitro and bone resorption in tissue culture and in vivo. Science. 1969 Sep 19;165(899):12624.

4. Fleisch H. Bisphosphonates: mechanisms of action. Endocr Rev. 1998 Feb;19(1):80-100.

5. Rodan GA. Mechanisms of action of bisphosphonates. Annu Rev Pharmacol Toxicol. 1998;38:375-88.

6. Lipton A, Theriault RL, Hortobagyi GN, Simeone J, Knight RD, Mellars K, et al. Pamidronate prevents skeletal complications and is effective palliative treatment in women with breast carcinoma and osteolytic bone metastases: long term follow-up of two randomized, placebo-controlled trials. Cancer. 2000 Mar 1;88(5):1082-90.

7. Berenson JR, Rosen LS, Howell A, Porter L, Coleman RE, Morley W, et al. Zoledronic acid reduces skeletal-related events in patients with osteolytic metastases. Cancer. 2001 Apr 1;91(7):1191-200.

8. Woo SB, Hellstein JW, Kalmar JR. Narrative [corrected] review: bisphosphonates and osteonecrosis of the jaws. Ann Intern Med. 2006 May 16;144(10):753-61.

9. Roelofs AJ, Thompson K, Gordon S, Rogers MJ. Molecular mechanisms of action of bisphosphonates: current status. Clin Cancer Res. 2006 Oct 15;12(20 Pt 2):6222s-30s.

10. Hughes DE, Macdonald BR, Russell RG, Gowen M. Inhibition of Osteoclasts-like cell formation by bisphosphonates the same proportion between medullar spaces and bone tissue.

\section{Acknowledgements}

This study was supported by a grant-in-aid from the São Paulo State Research Foundation (FAPESP) and a research postgraduate scholarship granted by the Brazilian Government Research Funding Agency CAPES. The authors are indebted to the Department of Physiology of Ribeirão Preto Dental School, University of São Paulo, Brazil, and to the histotechnician MS. Fátima Aparecida Silveira from the Service Pathology of Bauru Dental School, University of São Paulo, Brazil.

in long-term cultures of human bone marrow. J Clin Invest. 1989 Jun;83(6):1930-5.

11. Vitté C, Fleisch H, Guenther HL. Bisphosphonates induce osteoblasts to secrete an inhibitor of osteoclast-mediated resorption. Endocrinology. 1996 Jun;137(6):2324-33.

12. Anbinder AL, Prado FA, Prado MA, Balducci I, Rocha RF. The influence of ovariectomy, simvastatin and sodium alendronate on alveolar bone in rats. Braz Oral Res. 2007 JulSep;21(3):247-52.

13. Chaiamnuay S, Saag KG. Postmenopausal osteoporosis. What have we learned since introduction of bisphosphonates? Rev Endocr Metab Disord. 2006 Jun;7(1-2):101-12.

14. Farris EJ, Griffith JQ. The rat in laboratory investigation. $2^{\text {nd }}$ ed. Philadelphia: Lippincott Company; 1963. Chapter 1, Breeding of the rat;p. 1-17.

15. Keidel WD. Physiology. $2^{\text {nd }}$ ed. Barcelona: Salvat; 1971.

16. Ott SM. Clinical effects of bisphosphonates in involutional osteoporosis. J Bone Miner Res. 1993 Dec;8 Suppl 2:S597606.

17. Storm T, Steiniche T, Thamsborg G, Melsen F. Changes in bone histomorphometry after long-term treatment with intermittent, cyclic etidronate for postmenopausal osteoporosis. J Bone Miner Res. 1993 Feb; 8(2):199-208.

18. Boyce RW, Paddock CL, Gleason JR, Sletsema WK, Eriksen EF. The effects of risedronate on canine cancellous bone remodeling: three-dimensional kinetic reconstruction of the remodeling site. J Bone Minerv Res. 1995 Feb;10(2):211-21.

19. Russell RG, Rogers MJ. Bisphosphonates: from the laboratry to the clinic and back again. Bone. 1999 Jun; 25(1):97-106.

20. Van Beek E, Pieterman E, Cohen L, Löwik C, Papapoulos S. Farnesyl pyrophosphate synthase is the molecular target of 
nitrogen-containing bisphosphonates. Biochem Biophys Res Commun. 1999 Oct 14; 264(1):108-11.

21. Russell RG. Bisphosphonates: mode of action and pharmacology. Pediatrics. 2007 Mar;119 Suppl 2:S150-62.

22. Zhang FL, Casey PJ. Protein prenylation: molecular mechanisms and functional consequences. Annu Rev Biochem. 1996;65:241-69.

23. Zhang D, Udagawa N, Nakamura I, Murakami H, Saito S, Yamasaki K, et al. The small GTP-biding protein, Rho p21, is involved in bone resorption by regulating cytoskeletal organization in osteoclasts. J Cell Sci. 1995 Jun;108(Pt6):228592.

24. Fernandes MI, Gaio EJ, Oppermann RV, Rados PV, Rosing CK. Comparison of histometric and morphometric analyses of bone height in ligature-induced periodontitis in rats. Braz Oral Res. 2007 Jul-Sep;21(3):216-21.

25. Tani-Ishii N, Minamida G, Saitoh D, Chieda K, Omuro H, Sugaya A, et al. Inhibitory effects of incadronate on the pro- gression of rat experimental periodontitis by Porphyromonas gingivalis infection. J Periodontol. 2003 May; 74(5):603-9.

26. Weinreb M, Quartuccio H, Seedor JG, Aufdemorte TB, Brunsvold M, Chaves E, et al. Histomorphometrical analysis of the effects of the bisphosphonate alendronate on bone loss caused by experimental periodontitis in monkey. J Periodont Res. 1994 Jan;29(1):35-40.

27. Reddy MS, Weatherford TW 3rd, Smith CA, West BD, Jeffcoat MK, Jacks TM. Alendronate treatment of naturallyoccurring periodontitis in beagle dogs. J Periodontol. 1995 Mar;66(3):211-7.

28. Olson HM, Hagen A. Inhibition of post-extraction alveolar ridge resorption in rats by dichloromethane diphosphonate. J Periodontal Res. 1982 Nov;17(6):669-74.

29. Lepola VT, Hannuniemi R, Kippo K, Lauren L, Jalovaara P, Vaananen HK. Long-term effects of clodronate on growing rat bone. Bone. 1996 Feb; 18(2):191-6. Erratum in: Bone 1996 Aug; 19(2):205. 